\title{
由 由
} UNIVERSIDAD DEL PACÍFICO FACULTAD DE CIENCIAS EMPRESARIALES

\section{Motivaciones de los jóvenes emprendedores}

\author{
Alexis Boggio Salazar \\ Magíster en Administración por la Universidad del Piura, \\ Lima, Perú. Licenciado en Administración por la Universidad \\ del Pacífico. Profesor e investigador del Departamento \\ Académico de Ciencias Empresariales de la Universidad del \\ Pacífico, Lima, Perú. \\ Lorena Berrospi Castillo
}

\section{Resumen}

En la actualidad, tanto el emprendimiento como su gran impacto en el desarrollo de la sociedad son el foco de atención de un creciente campo de investigación, en particular de disciplinas como la economía, la psicología y la sociología. Desde que este tema es motivo de investigación, muchos estudios se han centrado principalmente en las características individuales de los emprendedores y en sus rasgos de personalidad. Sin embargo, en los últimos años la investigación sobre el emprendimiento ha cambiado de manera significativa. La orientación de los estudios fuerza una ampliación del campo y un aumento de su heterogeneidad a través de la aparición de nuevos temas cada vez más fascinantes y retadores. El objetivo de este estudio es describir las motivaciones o factores que inciden en el desarrollo de actividades de emprendimiento así como las percepciones de los emprendedores respecto de su idea y de las habilidades personales que detentaban para llevar a cabo su emprendimiento. En general, factores como la existencia de una oportunidad en el mercado, un mercado con atractivo comercial, un razonable conocimiento del mercado y la creencia de contar con una idea innovadora, son los factores más influyentes en la puesta en marcha de un emprendimiento. Los datos corresponden a una muestra de 165 egresados entre los años 1970 y 1995 de la Universidad del Pacífico.

\section{Palabras clave}

emprendimiento, emprendedor, emprendedorismo, entrepreneurship, entrepreneur, new venture. 


\section{Introducción}

Se puede decir que el emprendimiento es un fenómeno generalizado. Tanto en los países más ricos como en los más pobres, el emprendimiento es ampliamente considerado como una de las puertas que pueden permitir el acceso al crecimiento y a la prosperidad (Zahra, 1999). El emprendedor es parte de un complejo proceso por el cual las nuevas organizaciones inician su existencia: los emprendedores son esencialmente creadores de empresas (Kobia \& Sikalieh, 2010).

El emprendimiento es un campo de estudio retador y de mucha relevancia para académicos y ejecutivos. Es particularmente importante ya que puede contribuir a la prosperidad y el bienestar en diversos niveles: individual, familiar, comunitario, nacional e internacional. En este sentido, la ausencia del emprendimiento en el planteamiento de las teorías colectivas de los mercados, las empresas, las organizaciones y el cambio, hace que la comprensión que podamos tener del panorama empresarial sea incompleta (Shane \& Venkataraman, 2000).

Esta investigación in describe algunas de las razones por las que los jóvenes emprendedores decidieron iniciar un negocio propio y desarrollar su primera experiencia de emprendimiento empresarial.

\section{Objetivos del estudio}

- Conocer algunas de las motivaciones o factores más importantes que influyeron más en la ejecución o puesta en marcha de un emprendimiento.

- Conocer las percepciones de emprendedores respecto a ideas de negocios vinculadas a sus emprendimientos.

- Conocer las percepciones de los emprendedores respecto a sus capacidades personales vinculadas a sus emprendimientos.

\section{Marco conceptual}

Sin lugar a dudas, el riesgo asociado con el funcionamiento de un negocio tiene que ver con las habilidades del tomador de decisiones. Además, el riesgo con el que un gerente o emprendedor tiene que lidiar está asociado a su capacidad de control y a sus habilidades (Macko \& Tyszka, 2009). El emprendimiento se puede definir como el conjunto de esfuerzos para lograr un nuevo entorno económico, social, institucional y cultural a través de las acciones de un individuo o de un grupo de individuos (Rindova, Barry , \& Ketchen, Jr, 2009).

Es evidente que muchas de las definiciones del emprendimiento que se centran en la búsqueda de la creación de riqueza no son suficientes (Miller \& Collier, 2010). No obstante, para efectos de esta investigación utilizaremos, con el ánimo de definir un objetivo general, la definición de emprendimiento de Low \& MacMillan (1988): "creación de una nueva empresa". En este sentido, la investigación del emprendimiento trataría de explicar y facilitar el papel de la nueva empresa en la promoción del progreso económico.

Según Sánchez (2011) El campo del emprendimiento estaría localizado en la intersección de tres perspectivas: económica, sociológica y psicológica. (Figura 1)

Figura 1: Perspectivas del emprendimiento

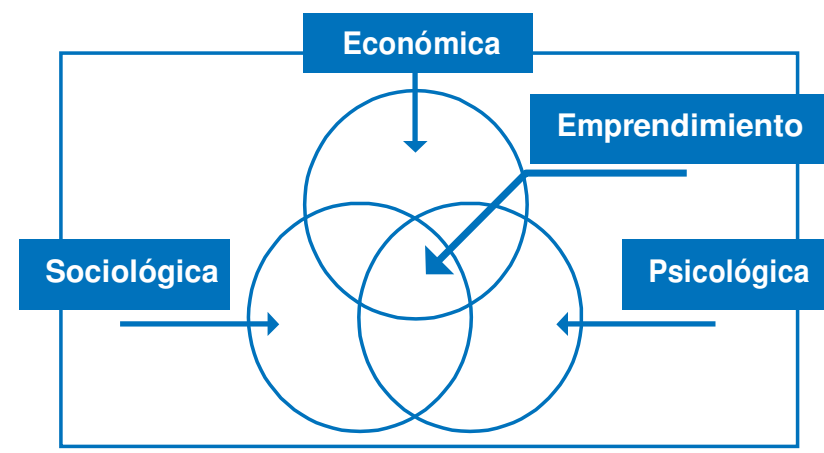

Fuente: (Sánchez, 2011)

La perspectiva psicológica abarca procesos cognitivos, rasgos de personalidad, factores genéticos. La perspectiva sociológica trata con redes sociales, factores socio-culturales e institucionales. La perspectiva económica está 
reflejada en costos de transacción, beneficios y efectos de la innovación como condiciones necesarias para el exitoso reconocimiento de oportunidades. (Figura 2)

Figura 2: Estructura teórica del emprendimiento

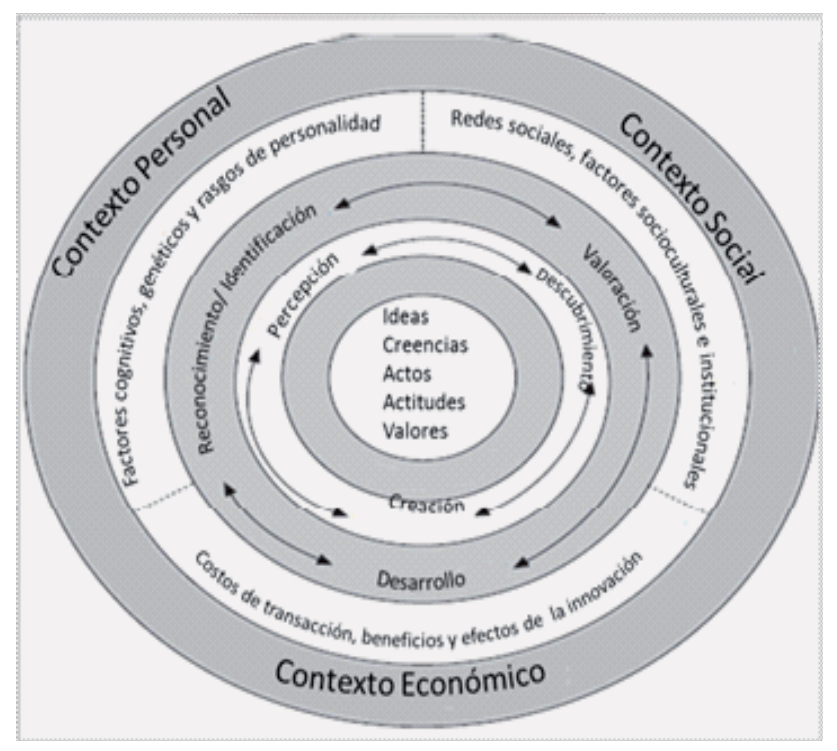

Fuente: (Sánchez, 2011)

Según Ucbasaran y otros (2001), se pueden distinguir los siguientes tipos de emprendedores: nacientes, principiantes, habituales, seriales y de portafolio.

- Emprendedores nacientes, esencialmente los que evalúan el establecimiento de un nuevo negocio.

- Los emprendedores principiantes son los que no tienen ninguna experiencia previa en la propiedad de un negocio, como podría ser el caso de un fundador de negocios, un heredero o un comprador de una empresa.

- Los emprendedores habituales, son aquellos con alguna experiencia previa en propiedad de las empresas.

- Los emprendedores seriales incluyen a aquellos que han vendido o cerrado su negocio original, pero posteriormente han heredado, establecido o adquirido otra empresa.

- En los emprendedores de portafolio se incluye a aquellas personas que han conservado su negocio original, pero que después de un tiempo han heredado, establecido o adquirido otra empresa.

\section{Motivaciones del emprendedor}

El contexto cultural en el cual las personas tienen su origen y desarrollo social desempeña un papel tremendamente influyente en la formación y modelación de los emprendedores, y en el grado en que ellos consideran el comportamiento emprendedor como algo deseable, como algo positivo (Morrison, 2000).

El papel de la familia, inmediata o extendida, es reconocido por tener el determinante potencial de hacer una contribución positiva hacia el comportamiento emprendedor a través de la provisión de modelos de rol inter-generacionales, y como proveedores de apoyo material e intangible (Morrison, 2000).

También es necesario considerar los modelos de roles ya que se vinculan con la idea de que conocer casos cercanos de emprendedores exitosos, hace que llegar a ser un emprendedor se vuelva mucho más creíble (Bygrave, 2004).

Por otro lado, no debe sorprender que gran parte del sustento de la mayoría de las actividades de emprendimiento se basan en la exploración, identificación y explotación de oportunidades (Schindehutte \& Morris, 2009).

Varios autores e investigadores han realizado estudios que justifican las acciones de emprendimiento, motivadas por factores a los que denominan motivaciones pull y push. (Block \& Wagner, 2007). La primera referida al concepto de oportunidad, y la segunda, al de necesidad.

Algunas motivaciones push y pull mencionadas a continuación:

\begin{tabular}{|l|l|}
\hline Motivaciones Push & Motivaciones Pull \\
\hline Ser autónomo & Gozar de reconocimiento social \\
Continuar con la tradición familiar & Desarrollar nuevos productos \\
Ser mi propio jefe & Desarrollar nuevos procesos de \\
producción \\
Salir del desempleo & $\begin{array}{l}\text { Ganar prestigio } \\
\text { Incrementar ingresos }\end{array}$ \\
\hline
\end{tabular}

Fuente: (Giacomin, 2007). 
Otros factores importantes, son La financiación e instrumentos financieros, la educación, la experienciaprofesional, aspectosdepersonalidad, conocimiento de capacidades propias, confianza en sí mismo.

\section{Formulación de ideas de negoco.}

Como ya se ha dicho, el proceso de emprendimiento se moldea por la intervención de distintos factores. Un individuo tiene una idea de un nuevo negocio y depende de los prospectos de carrera, familia, modelo de roles, estado de la economía, disponibilidad de recursos, perseguir esa idea. Casi siempre hay un "evento detonante" que permite el nacimiento de una nueva empresa. (Figura 3)

Figura 3: Modelo del proceso de emprendimiento:

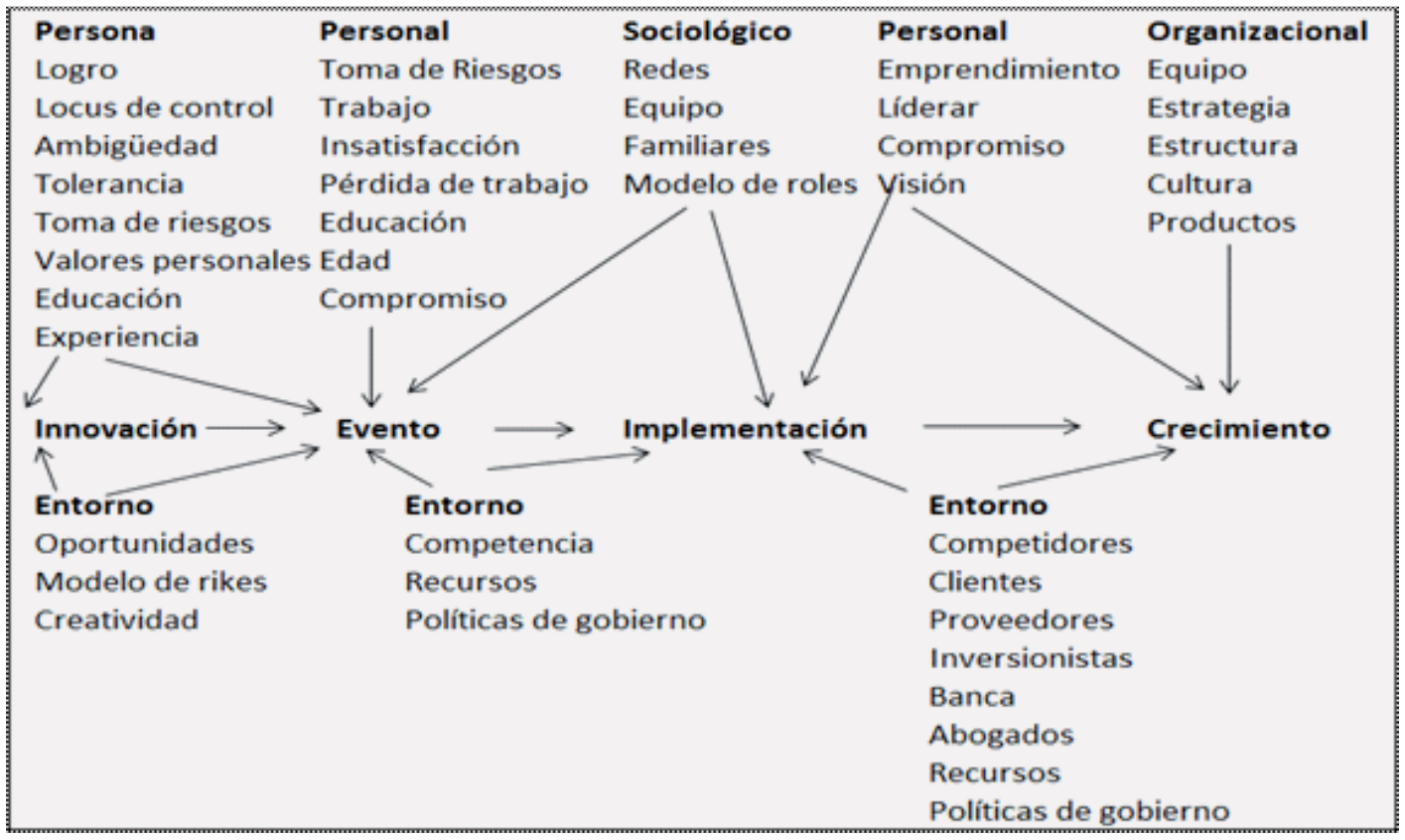

Fuente: Basado en Modelo de Carol Moore \& William D. Begrave presentado en "The Entrepreneurial Process"

\section{Competencias emprendedoras}

58 William Bygrave (2004) plantea una serie de características encontradas en la mayoría de emprendedores:

- Tienen visión a futuro de ellos y de sus negocios.

- Poseen una habilidad para implementar sus sueños.

- Pueden tomar decisiones con rapidez. Es la clave de su éxito.

- Una vez que toman una decisión, implementan la acción lo más rápido posible.

- Expresan un total compromisoa pesar de los obstáculos.
- Pueden estar totalmente dedicados a su negocio a pesar de las relaciones con sus amigos y familiares.

- Los emprendedores aman lo que hacen.

- Prestan gran atención a los detalles

- Quieren estar a cargo de su propio destino y no depender de un empleador.

- Consideran que si son exitosos serán retribuidos, pero no consideran que volverse adinerado es el principal motivador.

- Distribuyen la propiedad del negocio con los empleados claves para el éxito del mismo.

Para Fayolle (2002) un emprendedor es un gerente que se caracteriza por su capacidad 
para innovar, organizar y actuar (Identificando recursos, adquiriéndolos y disponiendo de ellos de la mejor forma posible para la producción). Los emprendedores detentan además una especial habilidad para liderar.

\section{Metodología}

\section{Método de muestreo}

En atención a los requerimientos de esta investigación se decidió utilizar la base de datos de los egresados brindada por la Oficina de Relaciones institucionales de la Universidad del Pacífico.

La muestra inicial se obtuvo de una base de 2198 egresados de todas las carreras de la Universidad del Pacífico entre los años 1970 y 1995.

Se utilizó un muestreo aleatorio por conglomerados y se eligieron las subpoblaciones del universo que se entregaron a los alumnos encargados de realizar la encuesta.

Para cada caso, el encuestador debía intentar primero ubicar a los encuestados con los datos disponibles en la base; sin embargo, muchos datos estaban desactualizados; por lo que se intentó llegar a los encuestados, en algunos casos, utilizando referencias.

Una vez ubicado el encuestado, se procedió a aplicar la encuesta personalmente. De no ser posible, la encuesta se aplicó vía skype, por teléfono y, en el peor de los casos, se envió el cuestionario vía e-mail para que sea autoaplicado por el encuestado.

Se realizó una supervisión y revisión de todas las encuestas recibidas y solo 164 de las 180 se encontraban aptas para utilizarse en la investigación.

La siguiente etapa consistió en la tabulación, elaboración de gráficos y análisis cualicuantitativo.

Los temas de la encuesta fueron:

1. Datos generales

2. Grado académico

3. Motivaciones

4. Ideas de negocio

5. Intención de ser parte de la red UP
Los temas más importantes están referidos a las motivaciones e ideas de negocio; sin embargo, hay que resaltar que la información utilizada sirvió también para actualizar los datos de los egresados que respondieron la encuesta.

\section{Diseño de cuestionario}

Las preguntas para los bloques 3 y 4 se evaluaron utilizando escalas Likert balanceadas y con opción neutra de cinco categorías, con puntuaciones que iban de 1 a 5.

Se hizo un análisis de perfil para cada reactivo de los 2 grupos más importantes de preguntas. Los resultados se presentan a continuación.

\section{Resultados}

\section{Motivaciones y factores más influyentes}

En los gráficos 1 al 9, se muestran las razones que más influyeron en la decisión de la puesta en marcha de algún negocio (para los 82 casos de egresados que iniciaron por lo menos una actividad de emprendimiento). A continuación el detalle de estos resultados:

Gráfico 1: Existencia de una oportunidad en el mercado

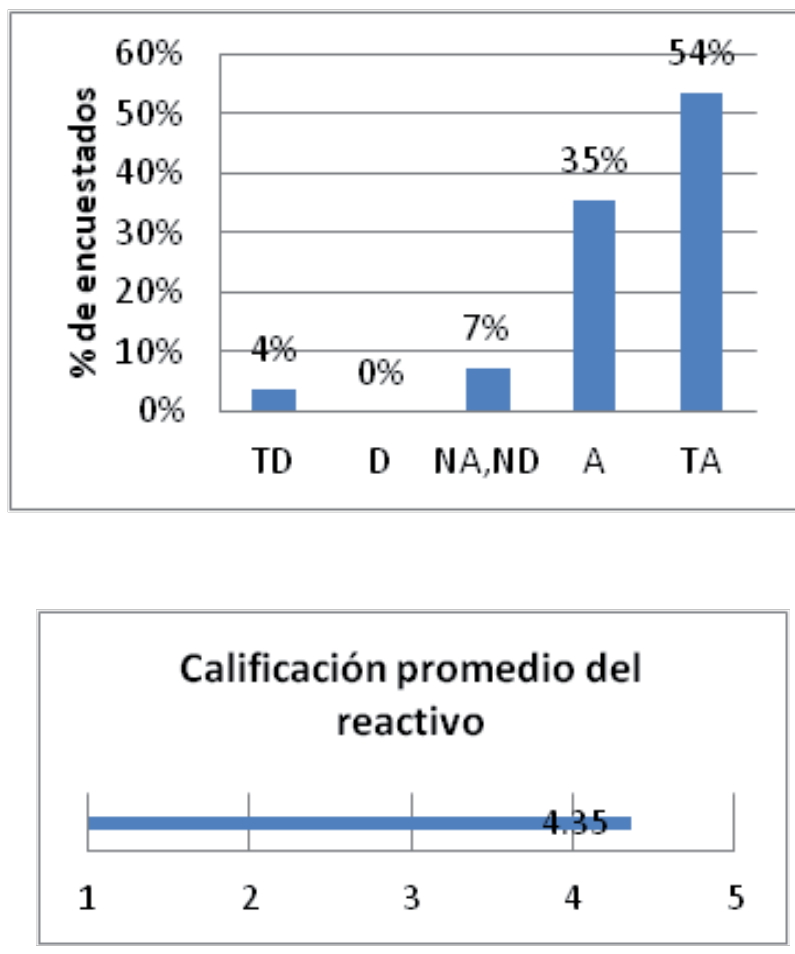


Según este gráfico, claramente el $89 \%$ de los encuestados manifestó haber estado influenciado a iniciar su emprendimiento por la existencia de una oportunidad en el mercado. Nótese que la categoría TA (totalmente de acuerdo) representó el $54 \%$ de las respuestas. Este reactivo obtuvo una puntuación de 4.35 en una escala con un máximo de 5 puntos. Este resultado no sorprende y de alguna manera apoya la creencia de que los emprendedores se motivan tanto por oportunidad como por necesidad.

Gráfico 2: Atractividad del mercado

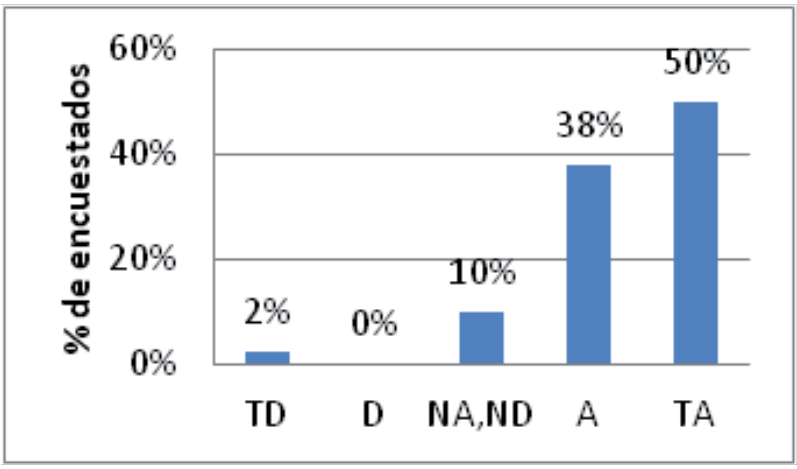

60

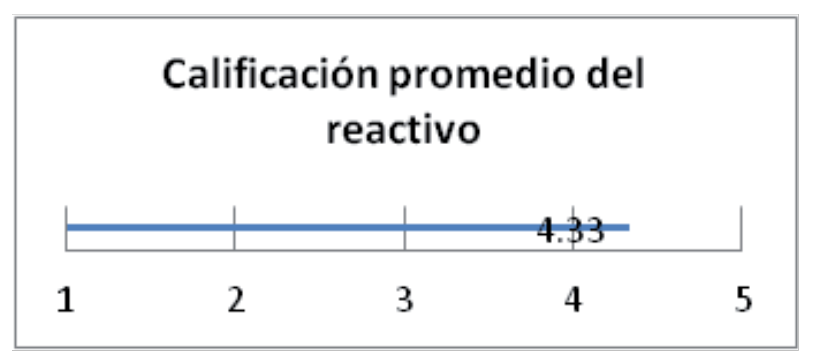

Cuando se propuso a los encuestados que "el mercado parecía atractivo", el 88\% mencionó estar de acuerdo o totalmente de acuerdo con este factor como un principal motivador para iniciar sus actividades emprendedoras. Muy en la línea con el reactivo anterior (existencia de una oportunidad), el $50 \%$ de los encuestados manifestaron estar totalmente de acuerdo con la afirmación, que tuvo una puntuación promedio de 4.33 .

Gráfico 3: Conocimiento del mercado
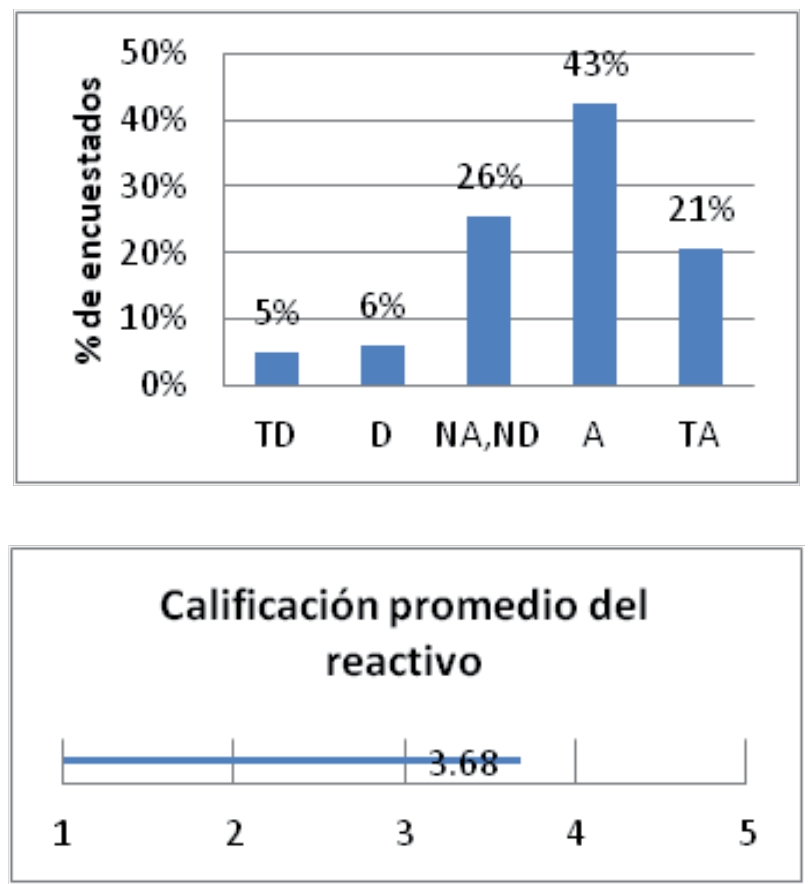

En el gráfico 3 se muestra que ante la afirmación "tenía un razonable conocimiento del mercado" las opiniones de los encuestados reflejan un acuerdo o total acuerdo del orden del $64 \%$. En este reactivo, sin embargo, $26 \%$ de los encuestados manifestaron estar ni de acuerdo ni en desacuerdo en que este factor fuera el que los motivó a iniciar su labor de emprendimiento. Una de las explicaciones que se pueden proponer para este resultado está en relación a la disposición al riesgo que es característico de los emprendedores. En promedio este reactivo obtuvo una calificación de 3.68 puntos.

Gráfico 4: Existencia de una idea innovadora

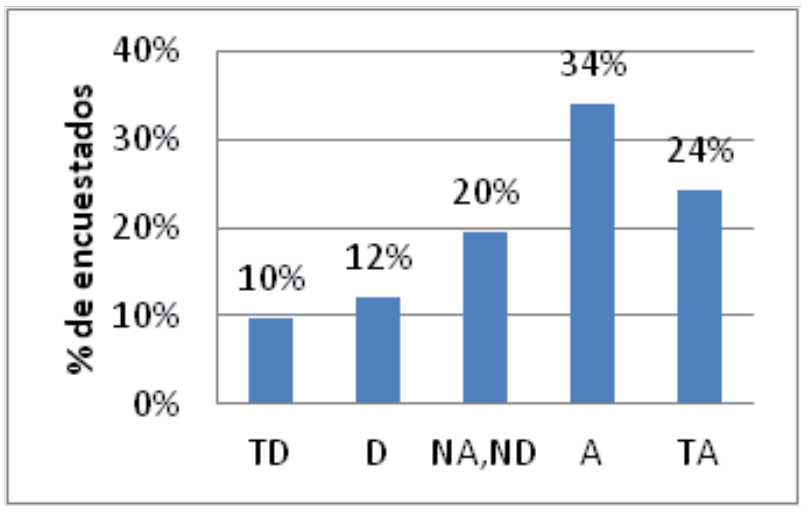




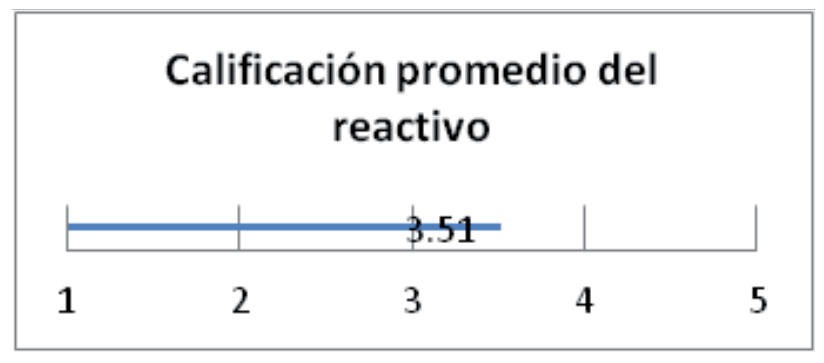

Según el gráfico 4, prácticamente un cuarto de los encuestados manifestaron que la idea de su emprendimiento no parecía innovadora al momento de ponerla en práctico. Este factor recibió $10 \%$ de respuestas en la categoría "totalmente en desacuerdo" y $12 \%$ en la categoría "en desacuerdo". Sin embargo, sigue siendo destacable que el $58 \%$ de los encuestados manifestaron estar "de acuerdo" o "totalmente de acuerdo". Estos resultados también van en la línea de que para los emprendedores la creencia de contar con una idea innovadora puede manifestarse como el detonante de la puesta en marcha de una aventura empresarial. La calificación promedio de este reactivo fue de 3.51 puntos.

Gráfico 5: Relación con la cadena de valor (proveedores, distribuidores, etc.).
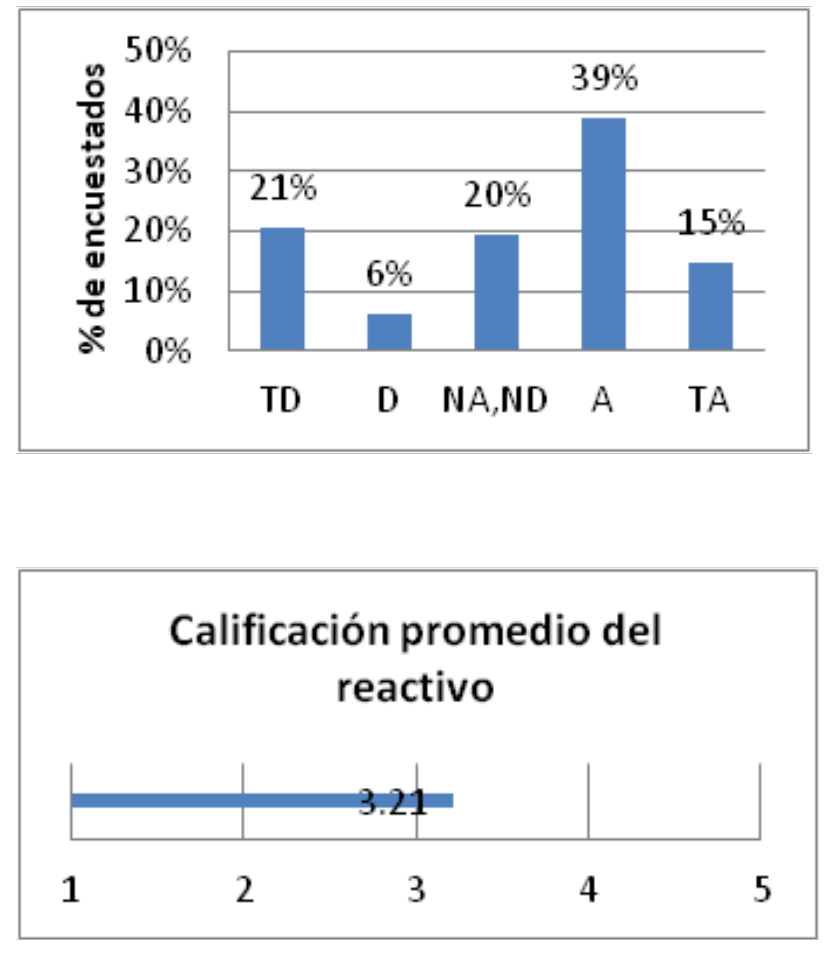

En el gráfico 5 se aprecian los resultados de las respuestas de los encuestados ante la afirmación “estabarelacionado conlacadenadevalor”.Según estos datos, este factor no era necesariamente una de las principales motivaciones para realizar un emprendimiento. Frente a esta afirmación los encuestados se manifestaron "de acuerdo" en un $39 \%$ y solo un $15 \%$ manifestó estar "totalmente de acuerdo". Este cuadro demuestra que en general los encuestados estaban más "totalmente en desacuerdo" que "totalmente de acuerdo" en el hecho de que hubieran relaciones relevantes con la cadena de valor: $21 \%$ totalmente en desacuerdo, $15 \%$ totalmente de acuerdo. Este factor alcanzó una calificación promedio de 3.21 puntos.

Gráfico 6: Facilidad de ejecución
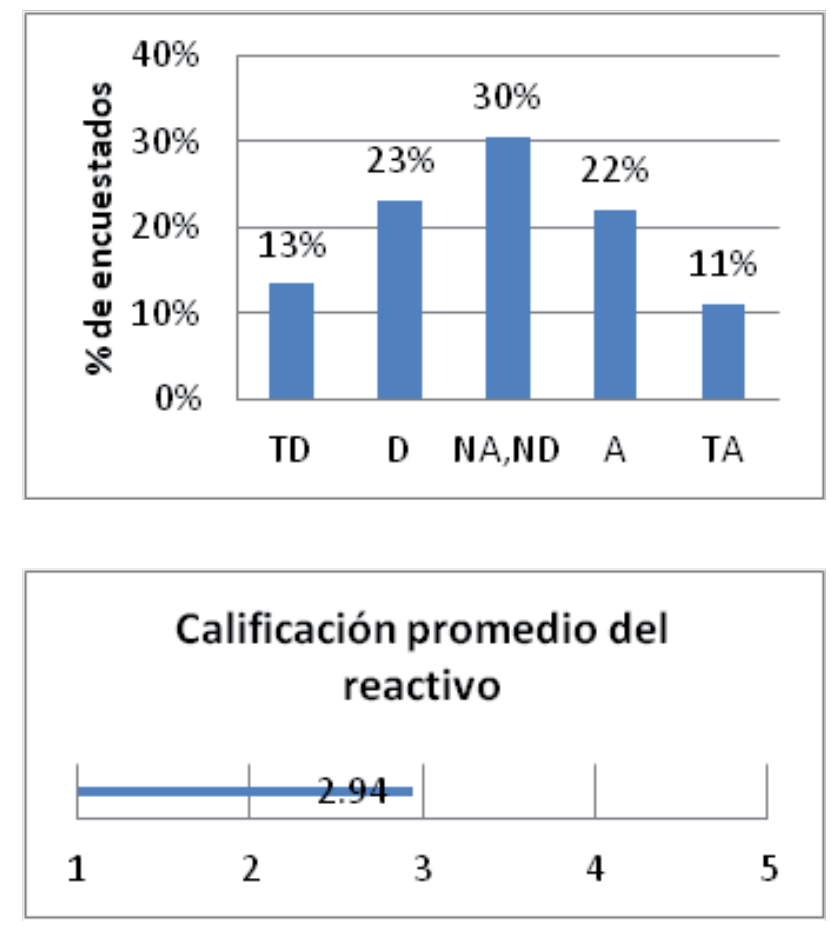

En el gráfico 6 se aprecia cuánto pude influir en los encuestados la percepción de que la idea será de fácil ejecución. Las respuestas que se obtuvieron con este reactivo son muy parejas y la mayoría de los encuestados (30\%) no estaba ni acuerdo ni desacuerdo con la afirmación. Sin embargo, el total de los encuestados que manifestó estar "totalmente en desacuerdo" o "en desacuerdo" alcanzó un nivel de $36 \%$, mientras que el total 
de los encuestados que manifestó estar "de acuerdo" o "totalmente de acuerdo" alcanzó un porcentaje de 33\%. Este reactivo obtuvo una calificación promedio de 2.94 puntos.

Gráfico 7: Contacto con inversionistas
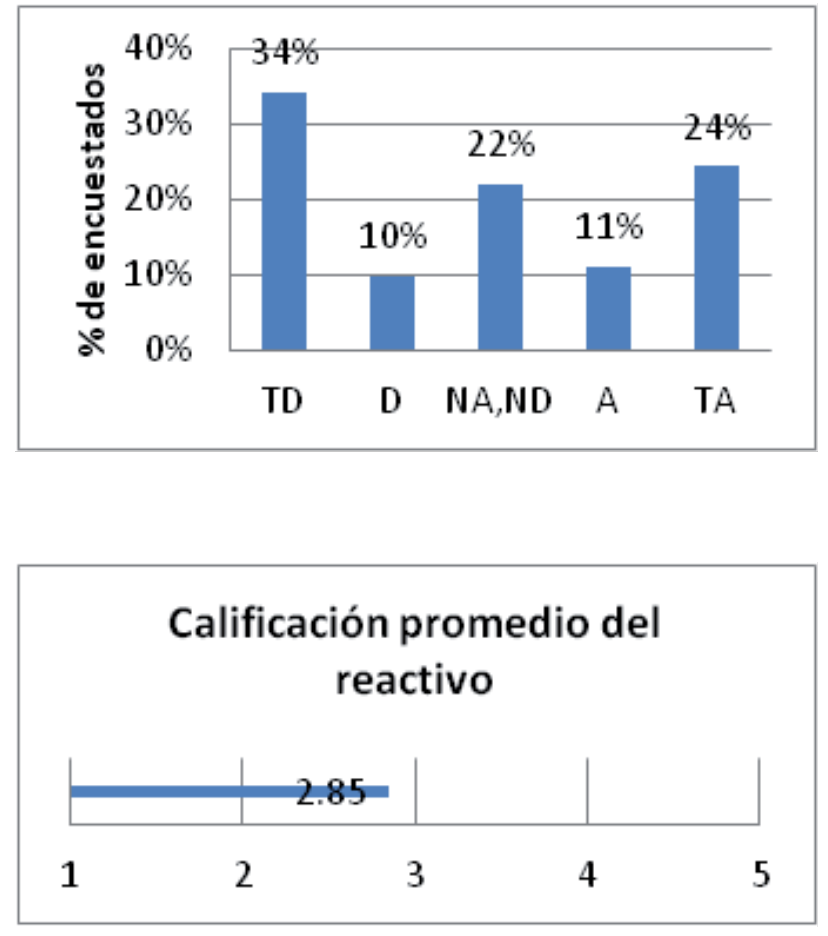

El gráfico 7 muestra la relevancia que tiene en opinión de los encuestados el haber tenido contactos con inversionistas interesados en su negocio. Según estos datos, es mayor la proporción de encuestados que manifestaron estar "totalmente en desacuerdo" o "en desacuerdo" (44\%) respecto a aquellos que manifestaron estar "de acuerdo" o "totalmente de acuerdo" (35\%). Se puede ver que la influencia de contactos con inversionistas para llevar a cabo un emprendimiento no es de los principales detonadores de las iniciativas del emprendedor. Esta afirmación tuvo una calificación promedio de 2.85 puntos.

Gráfico 8: Confianza en la idea de un amigo/ familiar

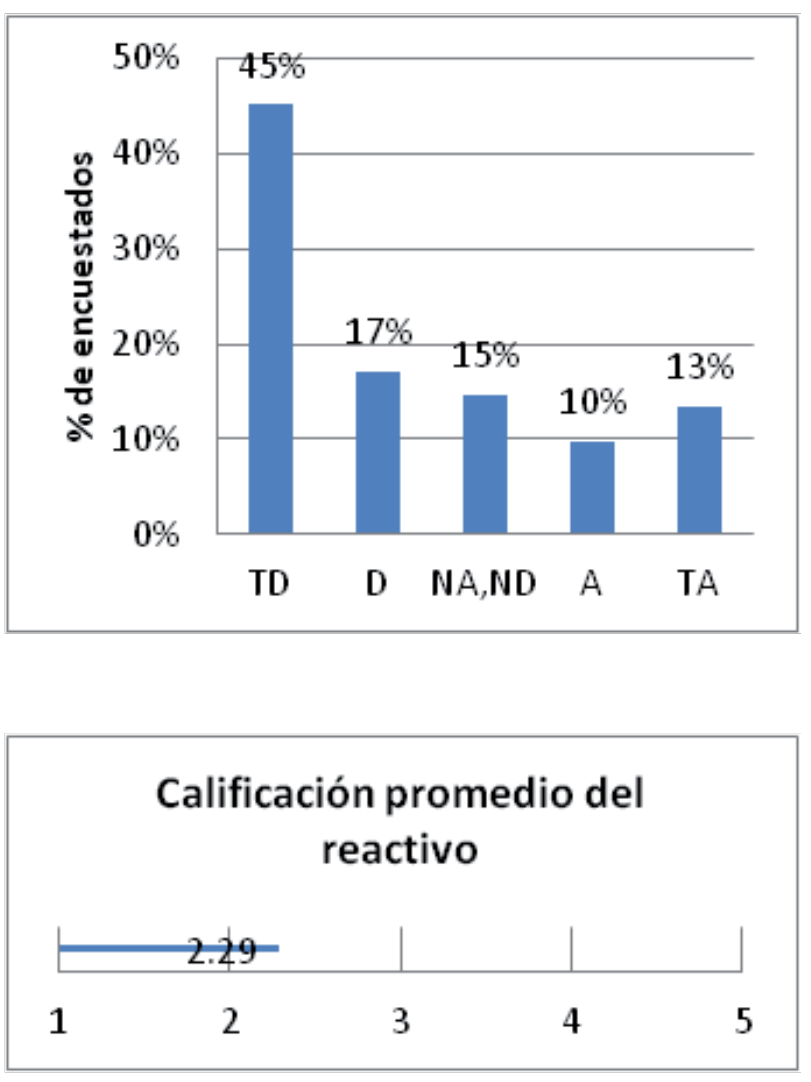

En la misma línea que el reactivo anterior, en el gráfico 8 se muestran los resultados que expresaron los encuestados frente a la afirmación "fue la idea de un amigo o familiar y confié en esa idea". Según los resultados, este factor es uno de los que menos aporta en las intenciones de emprender un negocio: nótese que el $62 \%$ de los encuestados se manifestaron como "totalmente en desacuerdo" o "en desacuerdo" frente a un pobre $23 \%$ de encuestados que manifestó estar "de acuerdo" o "totalmente de acuerdo" que consideraron este factor como influyente. Este reactivo obtuvo una calificación promedio de 2.29 puntos.

Gráfico 9: Activos de propiedad de un amigo/ familiar 

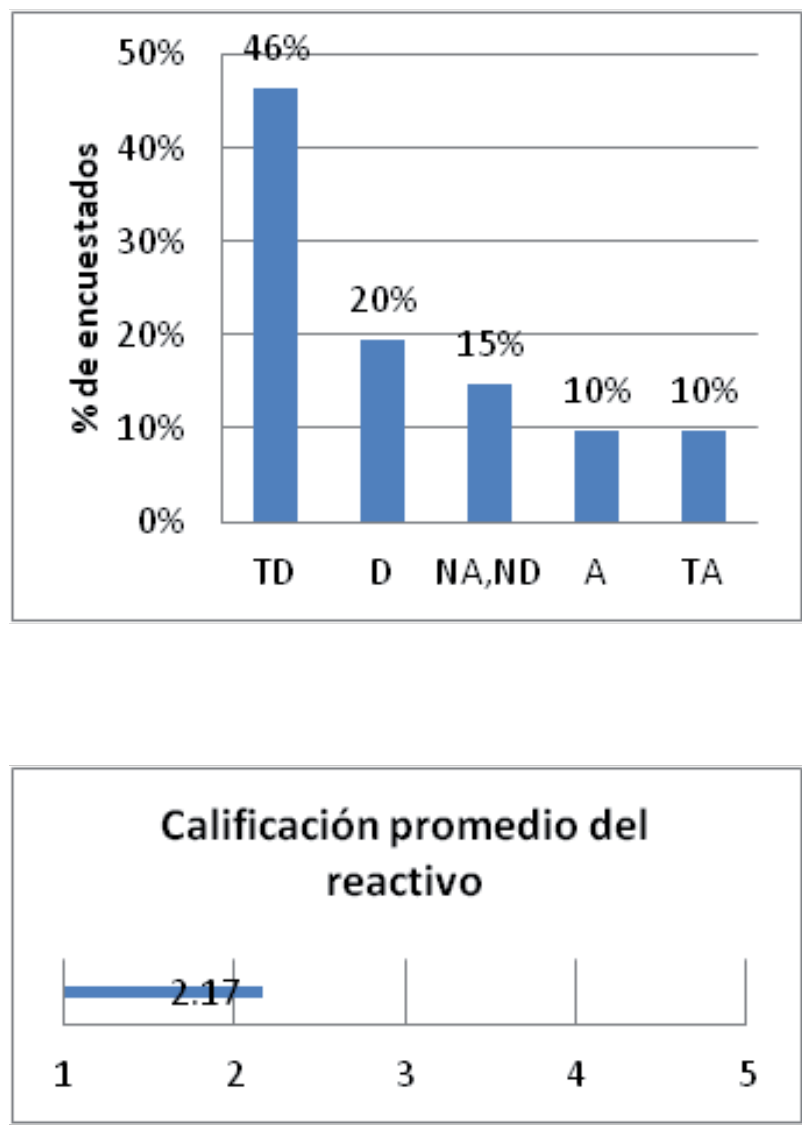

Finalmente, en el gráfico 9 se muestran las reacciones de los encuestados frente a la afirmación "un amigo o familiar poseía maquinarias, inmuebles, licencias, etc." Según los resultados, esta situación en opinión de los encuestados se presenta como el factor que menos pudo haber influido en las intenciones de emprendimiento de los egresados: la mayoría de los encuestados (66\%) expresó que estaba "totalmente en desacuerdo" o "en desacuerdo" con esta afirmación. Destacable también el hecho de que solo el $20 \%$ de los encuestados expresó estar "de acuerdo" o "totalmente de acuerdo" con la afirmación. Este reactivo alcanzó el menor puntaje promedio con 2.17 puntos, es decir, la mitad de lo que alcanzó el factor más influyente: existencia de una oportunidad en el mercado con una calificación promedio de 4.35 puntos.

Tabla 1: Ranking de la calificación

\begin{tabular}{|c|l|c|}
\hline & Motivaciones & $\begin{array}{c}\text { Calificación } \\
\text { promedio }\end{array}$ \\
\hline $1^{\circ}$ & Existencia de una oportunidad en el mercado & 4.35 \\
\hline $2^{\circ}$ & Atractividad del mercado & 4.33 \\
\hline $3^{\circ}$ & Conocimiento del mercado & 3.68 \\
\hline $4^{\circ}$ & Existencia de una idea innovadora & 3.51 \\
\hline $5^{\circ}$ & Relación con la cadena de valor & 3.21 \\
\hline $6^{\circ}$ & Facilidad de ejecución & 2.94 \\
\hline $7^{\circ}$ & Contacto con inversionistas interesados & 2.85 \\
\hline $8^{\circ}$ & Confianza en la idea de un amigo/ familiar & 2.29 \\
\hline $9^{\circ}$ & Activos de propiedad de un amigo/familiar & 2.17 \\
\hline
\end{tabular}

Base de encuestados: 82. Elaboración propia.

Gráfico 10: Ranking de la Calificación Promedio de los reactivos

En función a los resultados de este primer grupo de respuestas, se puede apreciar en el gráfico 10 que de los cuatro principales motivadores para iniciar una actividad emprendedora, los tres primeros están vinculados a factores relacionados al mercado (existencia de una oportunidad en el mercado, atractividad del mercado, conocimiento del mercado). El cuarto factor que se percibe como motivador para las intenciones de emprendimiento en orden importancia es la existencia de una idea innovadora.

De otro lado, los factores menos importantes que inciden en el hecho de iniciar una actividad de emprendimiento son: tener inversionistas interesados, la confianza en la idea de un amigo o familiar, y la disponibilidad de activos de amigos o familiares, este último, como ya se mencionó, recibió la menor calificación con un puntaje promedio de 2.17 sobre un total de 5 puntos. 


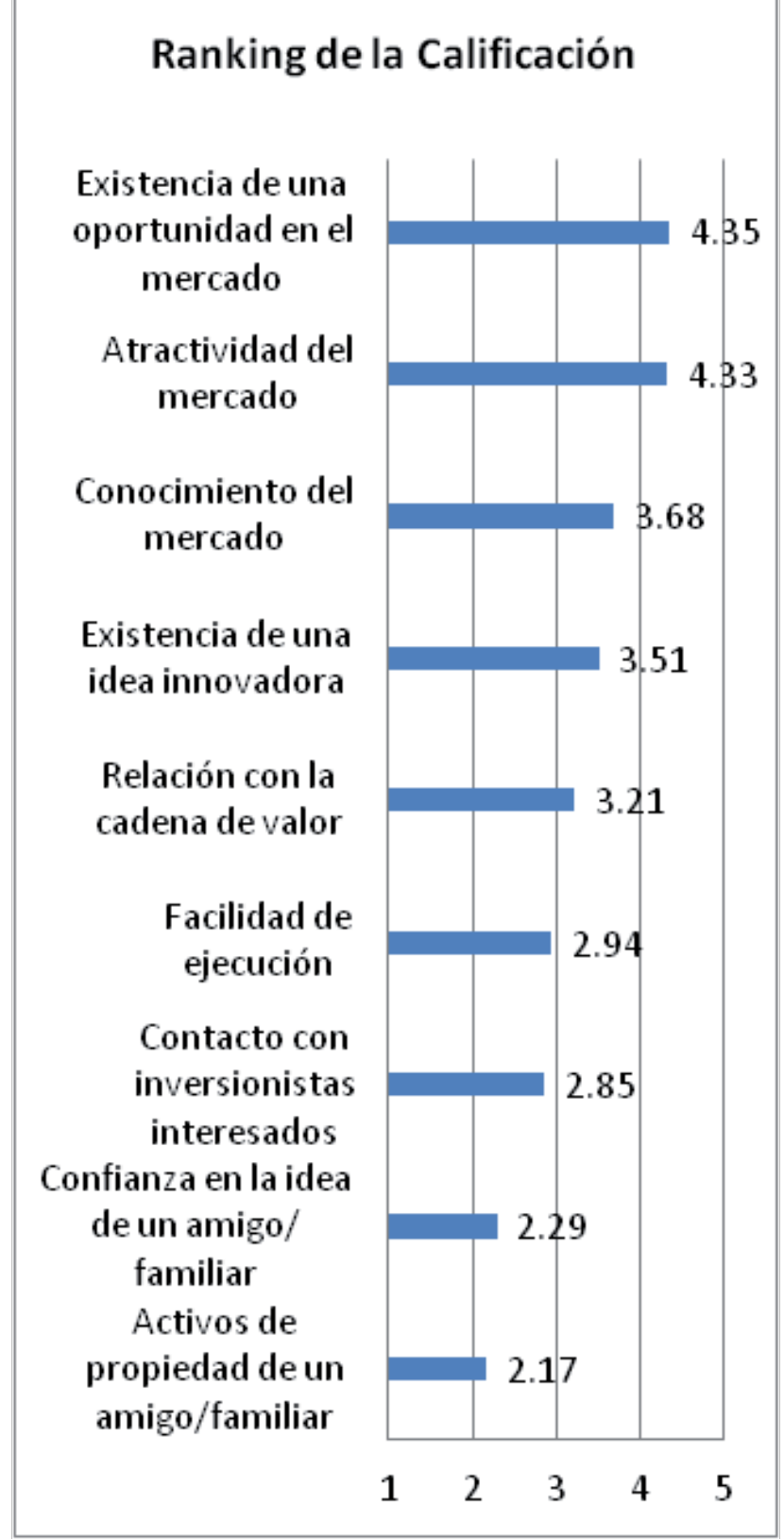

Formulación de ideas de negocios

Los gráficos 11 al 14 presentan los resultados relacionados con la evaluación de la idea de negocio (análisis apropiado de pros y contras, confianza en la viabilidad, carácter de innovadora).

Gráfico 11: Análisis de pros y contras
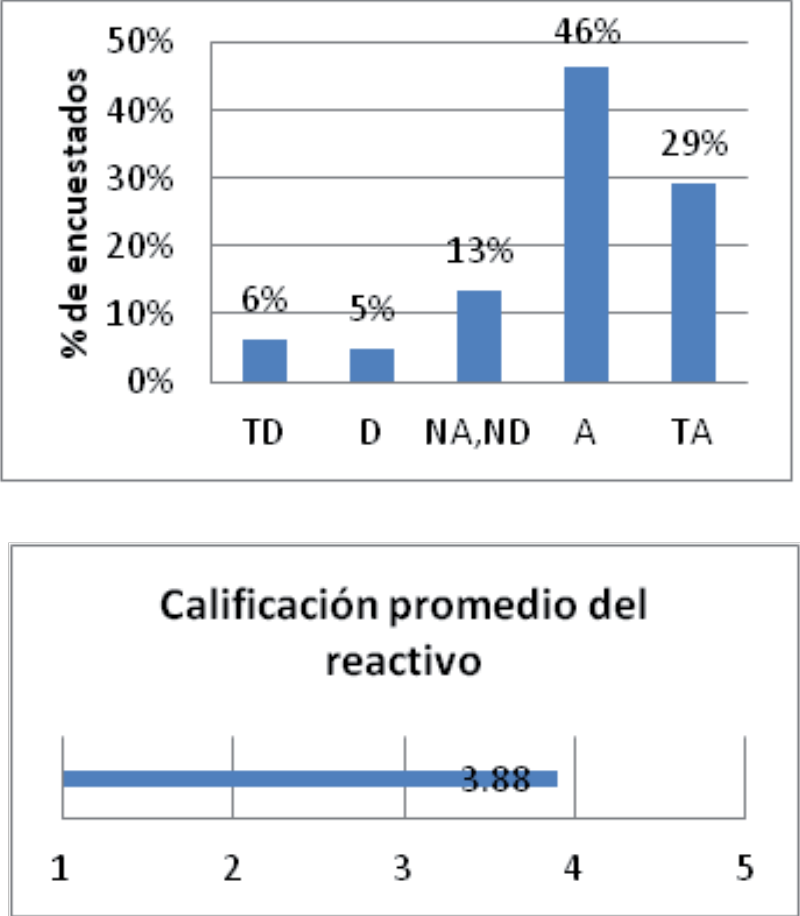

El gráfico 11 muestra que los encuestados en su mayoría manifiestan que realizaron un debido análisis de pros y contras (75\%) frente a un menor porcentaje que no realizó algún análisis $(11 \%)$. El puntaje promedio que recibió este reactivo fue de 3.88 .

Gráfico 12: Descarte de otras ideas de negocio antes de tomar la decisión

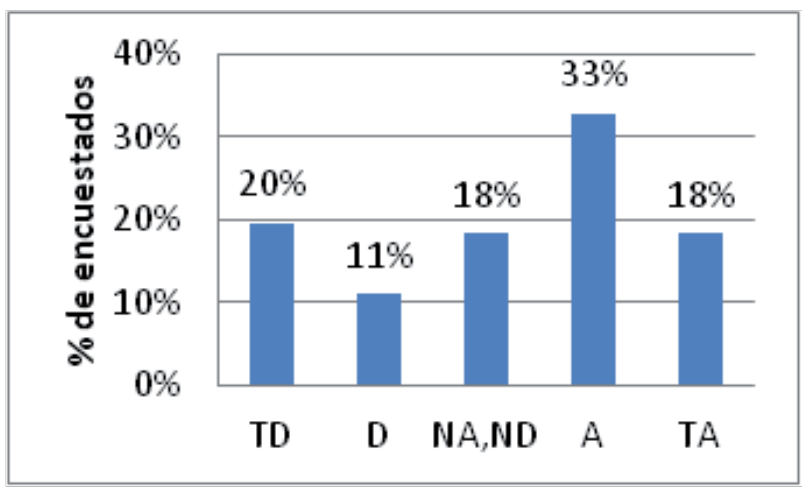

Calificación promedio del reactivo

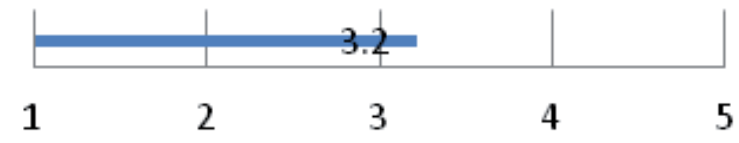


Según el gráfico 12 , el $51 \%$ de los encuestados manifestó que descartaron otras ideas de negocio antes de ejecutar el emprendimiento. El puntaje promedio de este reactivo fue de 3.2.

Gráfico 13: Viabilidad de la idea de negocio
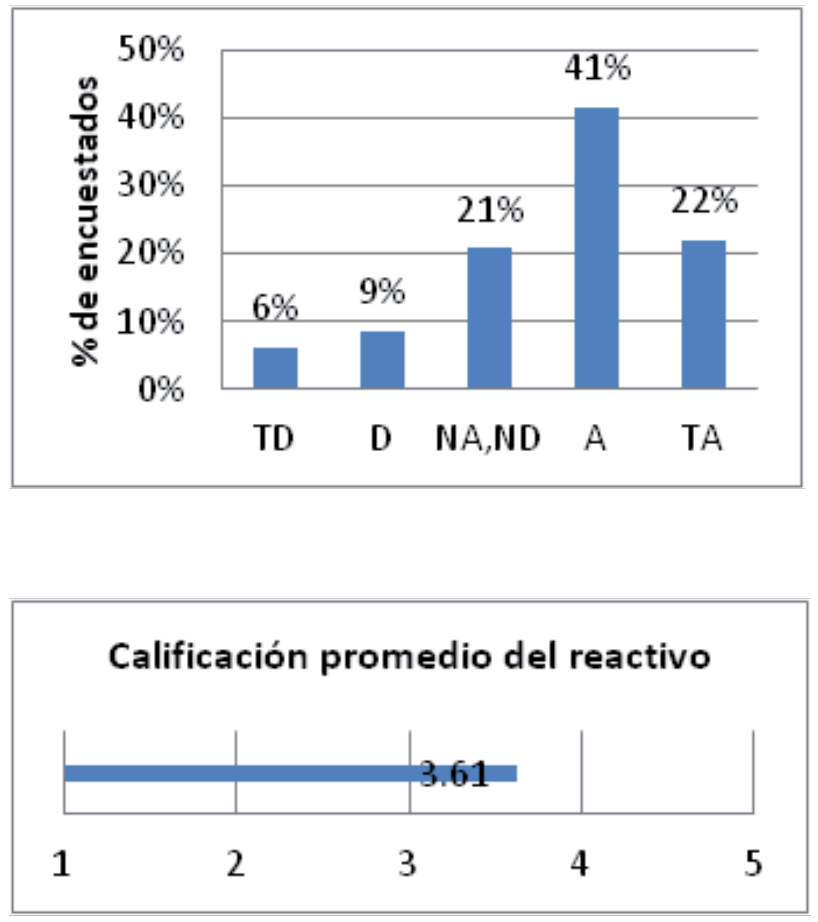

Respecto a la percepción de encuestados respecto a la viabilidad de su idea de negocio, la mayoría de los encuestados (63\%) manifestaron estar "de acuerdo" o "totalmente de acuerdo" con que su idea de negocio era viable. Solo el $15 \%$ de los encuestados manifestó que su idea no lo era, esto refleja una pequeña contradicción ya que uno podría esperar que en general los emprendedores confíen razonablemente en la viabilidad de su idea, al margen de lo complicado que pueda ser desarrollarla, los recursos que requiera, la experiencia del emprendedor, etc.

Gráfico 14: Carácter innovador de la idea de negocio
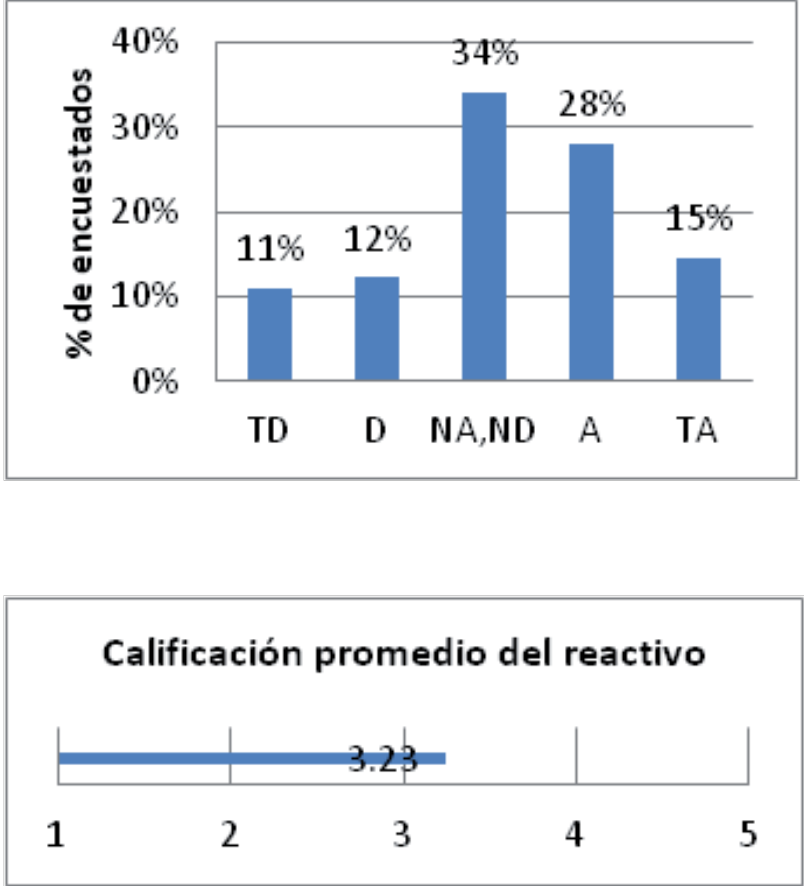

Para el gráfico 14, se muestran los resultados a la pregunta respecto al carácter innovador de la idea de negocio. En este caso, el porcentaje de encuestados que manifestaron estar "de acuerdo" o "totalmente de acuerdo" con este reactivo es menor: $43 \%$ frente al $58 \%$ que se obtuvo en el bloque anterior. El puntaje promedio de este reactivo fue de 3.23

\section{Competencias emprendedoras}

El segundo grupo tiene que ver con la autoevaluación de aspectos personales (percepción de capacidad para llevar a cabo un emprendimiento, adecuada investigación de mercados y explotación de redes de contactos). El tercer grupo incluye otros aspectos de autoevaluación como la percepción de errores cometidos en la elaboración del proyecto, la formación académica y el fomento de la Universidad del Pacífico para realizar actividades de emprendimiento.

Gráfico 15: Preparación y formación académica 

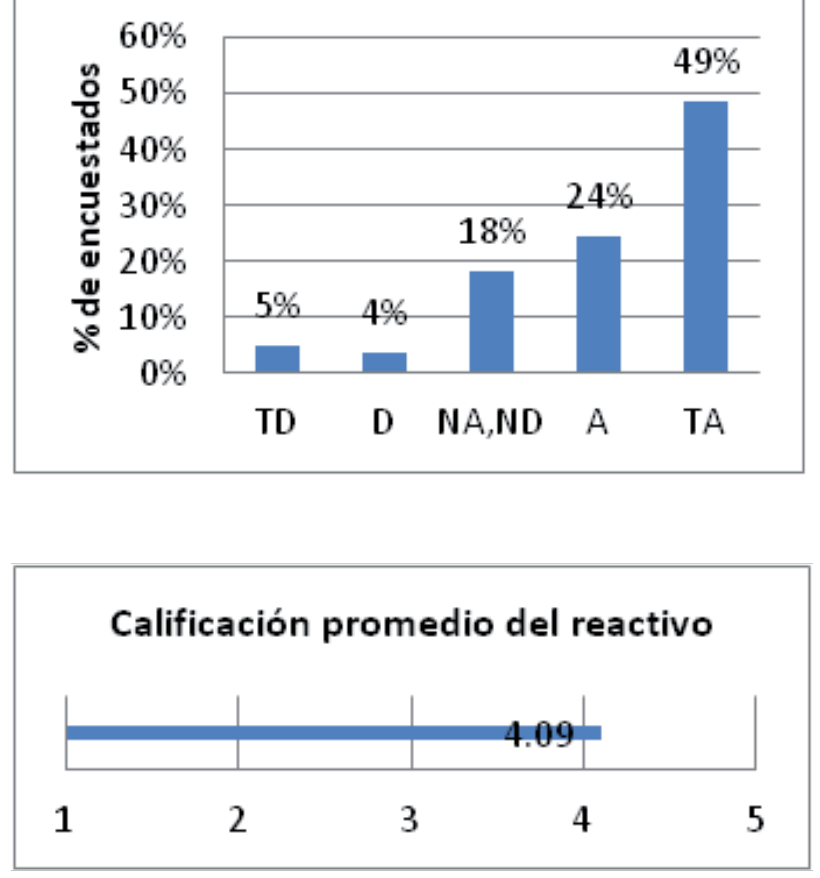

En el gráfico 15 se muestra la percepción de los egresados respecto a si se consideraban suficientemente preparados en lo académico al momento de llevar a cabo los temas propios de su emprendimiento. Según este gráfico un alto porcentaje de los egresados (73\%) manifestó estar "de acuerdo" o "totalmente de acuerdo". El cuadro también muestra una baja proporción de egresados que manifestaron estar en desacuerdo, total o parcialmente (9\%). Este reactivo obtuvo una calificación promedio de 4.09.

Gráfico 16:Deficiente realización de investigación de mercados

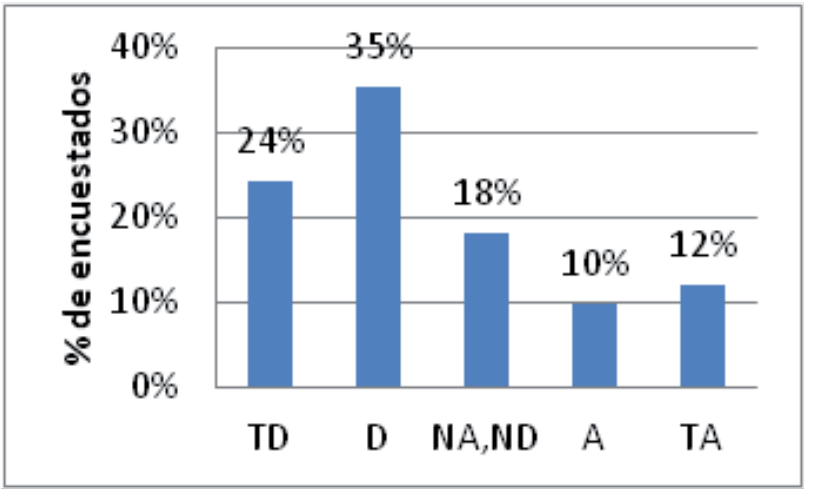

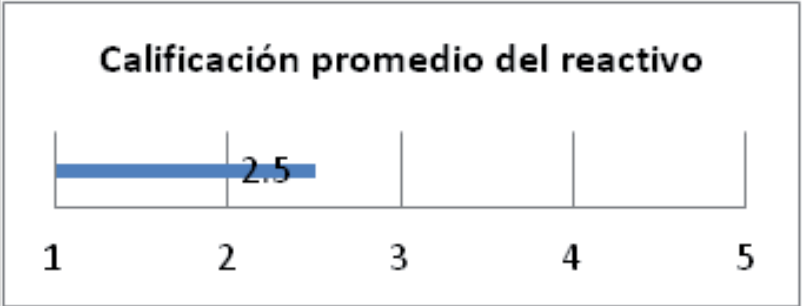

El gráfico 16 muestra que un considerable 59\% de los encuestados manifestó estar "totalmente en desacuerdo" o "en desacuerdo" respecto a la afirmación de haber realizado una mala investigación de mercados. Sin embargo, es revelador y algo preocupante que $22 \%$ de los encuestados sí consideren que la investigación de mercados que se llevó a cabo no fue satisfactoria.

Gráfico 17: Explotación de red de contactos
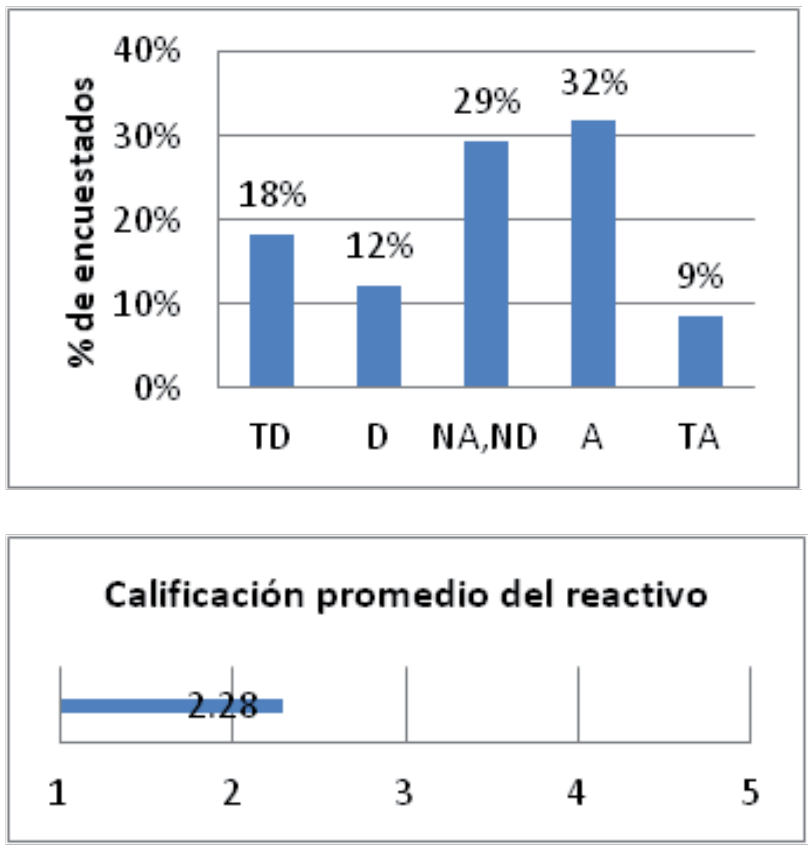

El gráfico 17 muestra la percepción de los encuestados respecto a los esfuerzos que pudieran haber realizado por aprovechar su red de contactos al momento de iniciar su emprendimiento. Los porcentajes muestran opiniones divididas frente a este reactivo, aunque es mayo la proporción de encuestados que manifestaron su conformidad con esta afirmación: $30 \%$ en desacuerdo vs. $41 \%$ de acuerdo.

Gráfico 18: Errores cometidos en la elaboración del proyecto. 
En el gráfico 18 se observa que, en general, la percepción de los egresados es que no cometieron mayores errores en sus emprendimientos (62\%) frente a un $18 \%$ que manifestó sí haberlos cometido. El puntaje promedio de este reactivo fue de 2.28.

\section{Conclusiones}

\section{Motivaciones}

La mayoría de los jóvenes emprendedores están muyinfluenciadosparainiciarunemprendimiento por la existencia de una oportunidad en el mercado. Este hecho no debe sorprender ya que se suele decir que los emprendedores se motivan tanto por oportunidad como por necesidad. La atractividad de mercado así como tener un razonable conocimiento de este también se presentan como motivadores para iniciar actividades emprendedoras. Para los emprendedores la creencia de contar con una idea innovadora puede manifestarse como el detonante de la puesta en marcha de una aventura empresarial. De otro lado, los factores menos importantes que inciden en el hecho de iniciar una actividad de emprendimiento tienen que ver con tener inversionistas interesados, la confianza en la idea de un amigo o familiar, y la disponibilidad de activos de amigos o familiares.

\section{Formulación de ideas de negocios}

Los jóvenes emprendedores en su mayoría manifiestan que realizaron un debido análisis de pros y contras y que descartaron otras ideas de negocio antes de ejecutar el emprendimiento, además coincidieron en que su idea de negocio era viable antes de ponerla en práctica. Respecto al carácter innovador de la idea de negocio, aunque es un factor importante, no parece ser determinante al momento de decidir si llevar a cabo o no un emprendimiento.

\section{Competencias emprendedoras}

Un alto porcentaje de los egresados se consideraban suficientemente preparados para llevar a cabo su emprendimiento; sin embargo, la mayoría coincidió en que se pudo haber realizado una más profunda investigación de mercados, paso que se considera muy importante al momento de desarrollar un plan de negocios. Los resultados también reflejan que el hecho de no haber aprovechado mejor su red de contactos es una autocrítica común a los jóvenes emprendedores. 


\section{BIBLIOGRAFÍA}

BLOCK, J., \& Wagner.

(2007). Opportunity recognition and exploitation by necessity and opportunity entrepreneurs: Empirical evidence from earnings equations, in: Solomon, George T. Sixty-Sixth Annual Meeting of the Academy of Management.

BYGRAVE, W. D.

(2004). The entrepreneurial process.

FAYOLLE, A. (December de 2002). Insights To Research On The Entrepreneurial Process From A Study On Perceptions Of Entrepreneurship And Entrepreneurs. Journal of Enterprising Culture, 10(4), 257-285.

GIACOMIN, O.

(2007). Novice creators: personal identity and push pull dynamics.

KOBIA, M., \& SIKALIEH, D.

(2010). Towards a search for the meaning of entrepreneurship, 34(2). Journal of European Industrial Training, 110-127.

LOW, M. B., \& MACMILLAN, I. C.

(1988). Entrepreneurship: Past Research and Future Challenges. Journal of Management, 14(2), 139-161.

MACKO, A., \& TYSZKA, T.

(2009). Entrepreneurship and Risk Taking. Applied Psychology: An International Review, 58(3), 469-487.

MILLER, R. A., \& Collier, E. W.

(2010). Redefining Entrepreneurship: A Virtues and Values Persspective. Journal of Leadership, Accountability and Ethics, 8(10), 80-89.
MORRISON, A.

(2000). Entrepreneurship: what triggers it? International Journal of Entrepreneurial Behaviour \& Research, 6(2), 59-71.

RINDOVA, V., Barry , D., \& Ketchen, Jr, D. J. (2009). Entrepreneuring as Emancipation. Academy of Management Review, 34(3).

SÁNCHEZ, J. C.

(2011). Entrepreneurship as a Legitimate Field of Knowledge. Psicothema, 23(3), 427-432.

SCHINDEHUTTE, M., \& MORRIS, M. H.

(2009). Advancing Strategic Entrepreneurship Research: The role of Complexity Science in Shifting the Paradigm. En Entrepreneurship: Theory \& Practice (págs. 241-276).

SHANE, S., \& VENKATARAMAN, S.

(2000). The promise of entrepreneurship as a field of research. Academy of Management Review, 25(1), 217-226.

UCBASARAN, D., WESTHEAD, P., \& WRIGHT, M.

(2001). The Focus of Entrepreneurial Research: Contextual and Process Issues. En Entrepreneurship: Theory \& Practice, 25(4) (págs. 57-80).

ZAHRA, S. A.

(1999). Academy of Management Executive, 13(1), 3642. 